Presented at the 4th European

Ri...

OCT 291986

\title{
SEMICONDUCTOR DETECTORS AND READOUT ELECTRONICS: PRESENT DIRECTIONS AND OUTSTANDING PROBLEMS*
}

v. Radeka

Brookhaven NatIonal Laboratory

Upton, New York 11973

\author{
September, 1986
}

*Th1s research was supported by the 0 . S. Department of Energy:

Contract No. DE-AC02-76CH00016.

By acceptance of this article, the publisher and/or recipient acknowledges the U. S. Government's right to retain a nonexclusive, royalty-free 11cense in and to any copyright covering this paper.

\section{DISCLAIMER}

This report was prepared as an account of work sponsored by an agency of the United States Government. Neither the Uniial States Government nor any agency thereof, nor any of their employees, makes any warranty, express or implied, or assurnes any legal liability or responsibility for the accuracy, compieteness, or usefulness of any information, apparatus, product, or process disclosed, or represents that its use would not infringe privately owned rights. Reference herein to any specific commercial product, process, or service by trade name, trademark, manufacturer, or otherwise does not necessarily constitute or imply its endorsement, recommendation, or favoring by the United States Government or any agency thereof. The views and opinions of authors expressed herein do not necessarily state or reflect those of the United States Government or any agency thereof. 


\section{SEMICONDUCTOR DETECTORS AND READOUT ELECTRONICS: PRESENT DIRECTIONS AND OUTSTANDING PROBLEMS \\ V. Radeka \\ Brookhaven National Laboratory, Upton, NY 11973 \\ An introduction to the Fourth European Symposium on Semiconductor Detectors, Munich, 3-5 March 1986}

The interest and activity in the development of semiconductor detectors with high position resolution has been increasing in the last six years. This activity has been stimulated by the need for high precision particle tracking in recent and proposed experiments in high energy physics and, to a lesser extent, by the need for $x$-ray position detection in some experiments with synchrotron radiation. The new developments and interest have resulted in a large number of publications and, sometimes, in a too high frequency of workshops and meetings.

To become familiar with the state of the art most rapidly, the best material to start wilh are the Proceedings of the Third European Symposium on Semiconductor Detectors, ${ }^{1}$ held in Munich in November of 1983 . The 1986 Munich Symposium approaches the subject in much greater depth as is apparent from this issue of the Proceedings. An excellent introduction to solid state vertex detectors has been provided by Damerell. 2

Most of the effort so far has been in the development of "microstrip" detectors with hybrid preamplifier readout. There are indications now that the emphasis is shifting toward readout integration as the most difficult problem. Microstrip detectors have been used in several high energy physics experiments. Not surprisingly, one finds that it is a long way

\footnotetext{
*This research was supported by the U. S. Department of Energy:
} Contract No. DE-ACO2-76CH00016.

By acceptance of this article, the publisher and/or recipient acknowledges the U. S. Government's right to retain a nonexclusive, royalty-free license in and to any copyright covering this paper. 
from a bench test of a detector to an accurate evaluation of its performance under the conditions of an experiment and of its benefit to the experiment. The most experience in this respect exists in connection with the experiment NA32 at the CERN SPS, and one can obtain an impression of the effort Involved from a report by Weilhammer. 3

The development of semiconductor position sensitive detectors for use in scientific experiments has been based on three main device concepts: microstrip (multi-diode) structures, charge coupled devices (CCDs) and drift devices. The first and the last of these have been concelved and developed outside of the semiconductor industry by the groups in research and university laboratories. CCDs have been developed in industrial laboratories for imaging in television and for military applications. Some spectal high performance devices have been developed for astrophysics by the industry. The use of CCDs in high energy physics has been limited to devices made for other purposes. ${ }^{2}, 4$ The drift device concept developed by Gatti and Rehak ${ }^{5}$ has also led them to propose a deep intion CCD. The development of a deep depletion $\mathrm{CCD}$ has been started by Struder et al. 6 Several different drift device configurations have been made by Kemmer and tested successfuily. ${ }^{7}$ One of these configurations, with cylindrical geometry and radial drift of electrons to a small anode, is of spectal interest since the charge is collected from a large area on a low capacitance electrode. This greatly reduces the amplifier noise contribution. Such a device is suitable as a photodiode for scintillation detectors and calorimeters and for large areas needed in ionization sampling calorimeters.

Experimental apparatus with silicon microstrip detectors provides a striking impression of the problems with interconnections and the size of the readout components. A microstrip detector with an area of $10-30 \mathrm{~cm}^{2}$ is 
surrounded by fan-out and preamplifier boards covering an area about two orders of magnitude larger. This is dictated by the size of the preamplifiers. The hybrid circuit preamplifiers presently used are an impressive technological achievement, and will remain a standard for comparison of noise of monolithic circuits. They were developed at several laboratories (BNL, CERN, Univ. Pavia, etc.) and some of them are available commercially. Microstrip detectors with hybrid preamplifiers and with interpolation with charge division among 2 to 4 strips - to keep the number of outputs at a minimum - are sat isfactory for fixed target experiments where space is available in the plane perpendicular to the beam. This space becomes restricted in some experiments, and the problem becomes severe to intractable in colliding beam experiments, or whenever a large solid angle has to be covered with detectors.

At low interaction rates, CCDs provide the best solution in principle with their good double track resolution. Their unambiguous position readout allows track reconstruction with fewer planes than with strip geometry, and makes it possible at high multiplicitiss. However, the area of available devices is just too small in some cases. Microstrip detectors and drift devices are presently made in larger areas than CCDs.

At high interaction rates and at not too ing multiplicities, microstrip detectors are still considered wost attractive in initial studies, before the readout questions are carefully considered. In most studies of future experiments with colliding beams, it is assumed that monolithic readout of strip detectors will be developed, and that the problems of amplifier size and of the number of output cables will disappear. It is usually stated that the readout problem is only one of Large Scale Integration (LSI). A closer look at this question reveals that if all the questions of processing technology are solved, we shall be faced 
with power dissipation as the most significant limitation. Power dissipation is not a technological question only, it is a question of fundamental limits. Certain voltage is required to operate an amplifying device, usually at least of the order of the bandgap of the semiconductor. The standing current in the device is determined from the transconductance and capacitance of the device required to obtain the minimum notse for a given capacitance of the detector. Thus the power dissipation of the input amplifying devise will depend on the detector capacitance. The power dissipation of the preamplifier and the whole readout channel will be many times that of the input device, and it will be inversely proportional to the required response time.

Prototypes of an LSI chip (known as MICROPLEX) developed by the CERN-HAWAII-STANFORD group have been tested recently. ${ }^{8}$ The power dissipation of this device is $\approx 17 \mathrm{~mW} /$ channel, and tt is just too high for continuous operation. The device is operated with the power switched on for a fraction of the time in synchronism with accelerator beam pulses. It is unlikely that less than $\approx 5 \mathrm{~mW} /$ channel can be achleved for complete readout for strip capacitances of up to $30 \mathrm{pF}$ and a response time in the 50 to 100 nsec range. Systems with $2 \times 10^{4}$ channels are contemplated for fixed target experiments, $2 \times 10^{5}$ channels for colliders (LEP, SLC and TEVATRON), and more than $10^{7}$ channels have been mentioned for the SSC. This would correspond to 100 watts, 1 kilowatt and 50 kilowatts: respectively. Obviously, the collider case needs more work.

The charge transfer principle (charge coupled devices) and the charge drift principle appear as more likely to provide a solution in the long term to the problem of a very large number of position resolution elements ("pixels") and of the power dissipation of readout amplifiers and signal processing. Present CCDs are considered "slow" because they were designed 
for imaging purposes with one readout channel for the whole device containing more than $10^{5}$ pixels, where all elements are read out sequentially. Obviously, CCDs could be designed with a number of readout channels (parallel-serial readout) as an optimum with respect to the readout time and power dissipation (in addition to deep depletion and a "fast clear" ${ }^{6}$ ). This approach is also not without probiems. A large detector system with CCDs requires voltages of 5-15 volts anc large currents propagating through the system at the charge transfer frequency (in the range of tens of megahertz), which leads to crosstalk and power dissipation.

The semiconductor drift device is a "quiet" device since only dc potentials are necessary for 1 ts operation. It can be made in larger areas $\left(20-30 \mathrm{~cm}^{2}\right)$ than present CCDs $\left(1-4 \mathrm{~cm}^{2}\right)$. It requires callbration because the carrier mobility is temperature-dependent. This is easily performed by charge injection from an auxiliary electrode. The readout electronics performs timing in addition to the charge measurement for centroid finding, which results in a somewhat higher power dissipation than for charge measurement on $1 y$. The charge repulsion and the diffusion of carriers produced by ionization determines the effective pixel size (i.e., the double track resolution). This depends on the drift velocity and it is between 200 and $400 \mu \mathrm{m}$, while the position resolution (accuracy) is similar to that of CCDs and microstrip detectors (in the range of a few micrometers). For track reconstruction close to the target (vertex finding), CCDs have an advantage since the diffusion during charge collection from even a deep depletion region is much smaller.

As the interaction rate increases (as contemplated for future hadron colliders) the compromise between the number of readout channels and the readout time tilts toward large numbers of readout channels, with a short 
readout time, where one enters the uncharted territory of powe dissipation, and high currents for charge transfer and/or signal multiplexing. It is apparent that all three device approaches show promise, have important applications and not negligible problems, so that a high level of activity on their development in the near future is warranted.

Radiation damage is one of the first questions raised when semfconductor detectors are considered for experiments at higher interaction rates. There is a certain amount of experience and understanding of fundamentals now to indicate that some microstrip structures and their hybrid circuit amplifiers are resistant enough, except at the highest luminosities contemplated. It is also fairly clear that in the development of monolithic solutions for detectors and readout amplifiers some processes and techniques will be preferable and some may have to be ruled out (e.g., junction devices are more resistant to radiations than MOS devices). An excellent introduction to radiation damage in semiconductor devices is provided by Van Lint. ${ }^{9}$ A detalled review of radiation damage in semiconductor detectors has been published by Kraner. ${ }^{10}$ Some very interesting effects of radiation on microstrip detectors are described by Klanner. 11

Monolithic integration of readcut electronics for semiconductor detectors will take two directions. One is integration of amplifiers and readout circuits separate fror the detector with bonding or some other type of connections to the detector. In this case it is difficult to reduce the capacitance below one or a few picofarads for the amplifier input device and the connection to the detector. The other dirertion will be development of detectors with amplifiers on the same wafer. In that case the capacitance of the charge collection electrode and the input amplifier device can be In the 50-100 femtofarad range. For strip (or pad) detectors wth a larger electrode capacitance the former solution is adequate, except 
for the difficulties with interconnections. Integration on the detector in such a case may be impractical considering also the variety of electrode configurations (strip lengths, interpolation factors, etc.) needed in different experiments. With small pixels, an important advantage of

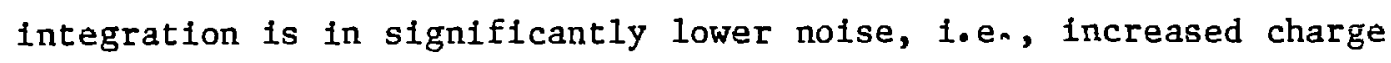
sensitivity. In considering various solutions and technologies versus the readout time, there are some limits which cannot be exceeded. The charge gain $G_{q}$ and the equivalent noise charge of an amplifying device are given by, 12

$$
\begin{aligned}
& \mathrm{G}_{\mathrm{q}}=(1 / 2) \mathrm{t}_{\mathrm{m}} / \tau_{\mathrm{A}}
\end{aligned}
$$

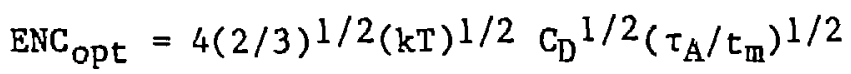

$$
\begin{aligned}
& =1.3 \times 10^{3} \mathrm{C}_{\mathrm{D}}^{1 / 2}[\mathrm{pF}]\left(\tau_{\mathrm{A}} / \mathrm{t}_{\mathrm{m}}\right)^{1 / 2} \text { [rms e] }
\end{aligned}
$$

where $\tau_{A}=C_{A} / g_{m}$ is the device time constant, $t_{m}$ is the measurement time (e.g., zero-to-peak time for a triangular or nearly triangular filter response such as a Gaussian), $C_{D}$ is the total detector electrode capacitance (with strays), the device capacitance $C_{A}$ equals the $C_{D}$.

The device time constant $\tau_{A}$ is also a measure of the carrier transit time $t_{e}$ through the channel (base) of the transistor. ( $\tau_{A}$ is in the range of 0.5 to $1 \mathrm{nsec}$ for discrete junction fleld-effect transistors at present.) It is obvious that fast devices with small dimensions in the direction of the current flow are preferred for low noise performance, and to achieve gain at short measurement times. Thus n-channel FETs are better than p-channel FETs. Yet p-channel junction devices can be more tasily realized with other ievices such as CMOS transistors. Junctiun. FETs are more resistant to radiation than MOS devices, and have much less excess noise. Bipolar transistors can uperate at lower power in amplifiers, but 
have higher noise due to the base current. MOS devices are sultable for switching and multiplexing. Therefore it is not 1ikely that any one device or any single process well established in integration of digital circuits will be suffictent if best performance with respect to noise and readout speed is required. There is some room for excess noise and nonoptimum performance with small area pixels, where the effect of amplifier noise is reduced by virtue of a low detector capacitance. There is not much margin with respect to noise for very long strips $(5-10 \mathrm{~cm})$ with capacitances in the 10-20 pF range. The semiconductor drift chamber is also quite demanding in that both low noise and fast response are required for good position resolution. Thus wa can expect that the combination of different processes or the new processes necessary for integration of the readouts alone, and particularly on the detector, wlll prove very challenging and will take some time.

Monolithic readout circuits will become useful and in some cases essential for other types of detectors used in high energy physics experiments, gas proportional and drift chambers, time projection chambers and calorineters. There are at least three different circuits of immediate interest:

1) Monolithic amplifiers for very fast gas proportional and drift chambers. In this application timing accuracy, speed of response and noise are critical. ${ }^{13}$ Bipolar transistor technology may be the best in this case because of large bandwidth and low power dissipation.

2) Monolithic preamplifiers, analog memory and multiplexed readout for gas drift and proportional chambers with cathode "pad" readout. Such a readout is needed for track reconstruction at very high particle multiplicities, and where accurate position information along anode wires is required. In this case a J-FET in the input is necessary for induced 
charge measurements, or an MOS FET if sufficiently low noise is achieved.

3) Monolithic preamplifiers (and other readout circuits) for liquid argon ionization chamber calorimeters. These circuits would have to operate in liquid argon (at $\approx 85^{\circ} \mathrm{K}$ ) in order to reduce the time required to transfer the charge from the chamber electrodes into the preamplifier, and in the case of a multiplexed readout, to reduce the number of interconnections through the cryostat. Present J-FETs have to be operated at $120-150^{\circ} \mathrm{K}$, since their noise is increased at $85^{\circ} \mathrm{K}$ due to impurity scattering. New devices have to be developed with a higher channel resistivity so that the noise minimum is shifted close to $85^{\circ} \mathrm{K}$.

The most difficult aspect of these and many other possible interesting developments based on monolithic technology is in high costs and low volume of devices to be used in research and therefore in the lack of incentives for the semiconductor industry to undertake the development and fabrication. Thus, principal development and design efforts 111 have to be at universities and research laboratories, while finding new ways to utilize semiconductor processing facilities provided by the industry. 


\section{References}

1. Proceedings of the Third European Symposium on Semiconductor Detectors, Munich, 14-16 November (1983) NIM 226 (1984) 1-218.

2. C. J. S. Damere11, "Developments in Solid State Vertex Detectors", RAL-84-123, (Lectures Presented at the SLAC Summer Institute, 1984).

3. P. Weilhammer, "Experience with Si Detectors in NA32", CERN-EP/86-54, (Presented at the Workshop "New Solid-State Devices for High Energy Physics", Berkeley, 28-30 October 1985).

4. C. J. S. Damere11, this issue.

5. E. Gatti and P. Rehak, NIM 225, (1984) 608.

6. L. Strüder, P. Ho11, J. Kemmer, and G. Lutz, this issue.

7. P. Rehak et al., NIM A248 (1986) 367.

8. R. Horisberget et al., this issue.

9. V. Van Lint, this issue.

10. H. W. Kraner, IEEE Trans. Nuc. Sci. NS-29 (1982) 1088 .

11. R. Klanner, this issue.

12. V. Radeka, NIM 226 (1984) 209.

13. J. Fischer, A. Hrisoho, V. Radeka, and P. Rehak, NIM A238 (1985) 249. 